\title{
Validação das equações que estimam a taxa metabólica de repouso em adolescentes meninas
}

\author{
Validation of the equations that estimate the \\ resting metabolic rate in adolescent girls
}

Paulo Henrique Santos da Fonseca', Maria de Fátima da Silva Duarte², Pedro Alberto Barbetta ${ }^{2}$

1 Universidade Estadual do Oeste do Paraná (Unioeste), Marechal Cândido Rondon, PR, Brasil 2 Universidade Federal de Santa Catarina (UFSC), Florianópolis, SC, Brasil
Correspondência para: Paulo Henrique Santos da Fonseca Av. Medianeira, 1.879 , ap. 603 97060-003 - Santa Maria, RS, Brasil paulo.phsf@gmail.com

Recebido em 8/Set/2008 Aceito em 20/Out/2009

\section{RESUMO}

Objetivos: $O$ objetivo deste estudo é analisar a validade das equações de predição da taxa metabólica de repouso (TMR) propostas por Harris e Benedicti (1919), Schofield (1985), WHO/FAO/UNU (1985), Henry e Rees (1991), Molnár e cols. (1995), Tverskaya e cols. (1998) e Müller e cols. (2004) em adolescentes meninas. Sujeitos e métodos: Para tanto, avaliaram-se 51 meninas entre 10 e 17 anos, sendo mensuradas as seguintes variáveis: massa corporal, estatura, massa gorda (absoluta e percentual) e massa magra (absoluta e percentual), bem como os valores do consumo de $\mathrm{VO}_{2} \mathrm{e}$ produção de $\mathrm{VCO}_{2}$ que foram substituídos na equação de Weir (1949) considerada, neste estudo, como método padrão daTMR. Realizaram-se os seguintes procedimentos estatísticos: teste " $\mathrm{t}$ " pareado $(p<0,05)$; erro constante $(E C)$ com diferença menor que $5 \%$ e análise gráfica de Bland e Altman (1986). Resultados: As equações propostas por Tverskaya e cols. (1998) e Müller e cols. (2004) foram as únicas que apresentaram diferenças significativas entre as médias. No entanto, somente as equações propostas por Henry e Rees (1991) e Molnár e cols. (1995) obtiveram erro constante inferior a 5\%. Porém, as análises gráficas de Bland e Altman (1986) revelaram que, independentemente da equação, não há concordância entre as equações e o método padrão. Conclusões: $O$ estudo indicou que as equações não aprovaram nos critérios estipulados e que, portanto, não possuem validade no cálculo daTMR. Arq Bras Endocrinol Metab. 2010;54(1):30-6

\section{Descritores}

Gasto energético; taxa metabólica de repouso; saúde; adolescentes

\section{ABSTRACT}

Objectives: The objective of the study is to analyze the validity of the prediction equations for the resting metabolic rate (RMR) proposed by Harris and Benedicti (1919), Schofield (1985), WHO/FAO/ UNU (1985), Henry and Rees (1991), Molnár and cols. (1995), Tverskaya and cols. (1998) and Müller and cols. (2004) in adolescent girls (51 girls between 10 and 17 years of age). Subjects and methods: The following anthropometric variables were measured: body weight, stature, body fat mass (absolute and percentage), body lean mass (absolute and percentage) as well as the consumption of $\mathrm{VO}_{2}$ and production of $\mathrm{VCO}_{2}$ that were substituted in Weir's (1949) equation which was considered the RMR standard method in this study. The following statistical procedures were carried out: paired t-test $(p<0.05)$; constant error (CE) with a less than $5 \%$ difference and Bland and Altman's (1986) graphical analysis. Results: The equations proposed by Tverskaya and cols. (1998) and Müller and cols. (2004) were the only equations that presented significant differences between the averages. However, only the equations proposed by Henry and Rees (1991) and Molnár and cols. (1995) produced a constant error of less than $5 \%$. However, Bland and Altman's (1986) graphical analysis showed that independently of the equation there is no agreement between the equations and the standard method. Conclusions: The study indicates that none of the equations meet all the stipulated criteria and that thus have no validity in the calculation of the RMR. Arq Bras Endocrinol Metab. 2010;54(1):30-6

Keywords

Energy expenditure; resting metabolic rate; health; adolescents 


\section{INTRODUÇÃO}

$\mathrm{A}^{\mathrm{i}}$ intervenção para minimizar o surgimento de problemas de saúde contemporâneos, como doenças coronarianas, obesidade, hipertensão, osteoporose, entre outras, está associada à capacidade de se conhecer o gasto energético diário dos indivíduos.

Há um consenso na literatura $(1,2)$ de que, nos seres humanos, a energia diária é gasta por meio de três maneiras: pela taxa metabólica de repouso (TMR), pelo efeito térmico dos alimentos e pela atividade física muscular. $\mathrm{Na}$ infância e na adolescência, sugere-se o acréscimo de mais um elemento: o anabolismo/crescimento (3).

Considera-se que a TMR é definida como a taxa mínima de energia consumida necessária para sustentar todas as funções celulares e que responde por $60 \%$ a $70 \%$ do total de gasto energético diário nos humanos (4). A TMR representa o principal componente do gasto energético total (5), especialmente quando se determina a necessidade diária de energia em pessoas sedentárias (6).

A TMR tem sido utilizada rotineiramente por clínicas para estimar as necessidades energéticas em pacientes, por agências governamentais e por organizações de saúde para definir as necessidades energéticas da população (4) e, também, no esporte, para a orientação energética em atletas (7).

Subestimar ou superestimar a TMR pode gerar um erro na medida do cálculo de energia para um indivíduo ou para populações. Dessa forma, a medida correta é importante para uma indicação mais precisa das necessidades energéticas (8), determinando com maior eficiência os planos de nutrição $(9,10)$, motivo que justifica o fato de o uso contínuo de equações ser reexaminado (11).

Recentes pesquisas internacionais $(12,13)$ e nacionais $(14,15)$ estão sendo conduzidas para desenvolver e validar equações que estimam a TMR. No Brasil, es- tudos realizados apontam ser imperativo que equações apropriadas sejam desenvolvidas para a nossa população $(5,14,16)$.

Dessa forma, o presente estudo tem como objetivo analisar a validade das equações de predição (Tabela 1) da TMR em adolescentes meninas.

\section{SUJEITOS E MÉTODOS}

A presente pesquisa e o termo de consentimento livre e esclarecido foram aprovados pelo Comitê de Ética em Pesquisa da Universidade Federal de Santa Catarina (UFSC) e estão registrados pelo número 091/06.

A amostra foi constituída de 51 meninas com idade entre 10 e 17 anos, segundo a faixa etária sugerida pela WHO (19), e classificada pelo índice de massa corporal (23) em baixo peso, peso adequado, sobrepeso e obeso, como ilustra a tabela 2 . Os indivíduos que participaram da pesquisa foram escolhidos ao acaso, não havendo cálculo estatístico para determinação do número de participantes.

As avaliações ocorreram no Laboratório de Esforço Físico (LAEF) da UFSC sempre no período da manhã e foram no máximo quatro sujeitos avaliados por manhã.

No dia da avaliação, foram apresentados os termos de consentimento livre e esclarecido aos pais e adolescentes e o protocolo do Comitê de Ética em Pesquisa, que esclarece que o estudo não coloca em risco a integridade física e psicológica dos indivíduos estudados.

A avaliação do LAEF seguiu os seguintes passos: primeiramente, o sujeito respondeu a uma anamnese. As adolescentes que não conseguiram responder às questões receberam auxílio dos pais e pesquisadores. Depois, foram realizadas as avaliações da massa corporal (Balança Plenna Digital, modelo MEA-03140, USA), estatura (Balança Toledo, modelo 2096 PP, BR), além

Tabela 1. Equações que estimam a TMR de adolescentes meninas

\begin{tabular}{|c|c|c|c|c|}
\hline Autor & $\mathbf{N}$ & Faixa etária & Equação & $\mathbf{R}^{2}$ \\
\hline Harris e Benedict (17) & 103 & $15-74$ & $\mathrm{kcal} / \mathrm{d}=655,0955+9,5634^{*}(\mathrm{MC})+1,8496^{\star}(\mathrm{ET})-4,6756$ (ID) & 0,53 \\
\hline Schofield (18) & 575 & $10-17$ & $\mathrm{MJ} / \mathrm{d}=0,056^{\star}(\mathrm{MC})+2,898$ & 0,80 \\
\hline WHO (19) & - & $10-17$ & $\mathrm{MJ} / \mathrm{d}=0,0510^{*}(\mathrm{MC})+3,12$ & 0,56 \\
\hline Henry e Rees (20) & 233 & $10-17$ & $\mathrm{MJ} / \mathrm{d}=0,047^{\star}(\mathrm{MC})+2,951$ & 0,39 \\
\hline Molnár e cols. (21) & & & $\mathrm{kJ} / \mathrm{d}=51,2^{\star}(\mathrm{MC})+24,5^{*}(\mathrm{ET})-207,5^{\star}(\mathrm{ID})+1629,8$ & 0,82 \\
\hline Tverskaya e cols. (22) & 110 & $6-18$ & $\mathrm{kcal} / \mathrm{d}=775+28,4^{*}(\mathrm{MCM})-37^{*}(\mathrm{ID})+3,3^{*}(\mathrm{MCG})+82^{*}(\mathrm{~S})$ & 0,84 \\
\hline Müller e cols. (13) & 423 & $5-17$ & $\begin{array}{c}\mathrm{mJ} / \mathrm{d}=0,02606^{*}(\mathrm{MC})+0,04129^{*}(\mathrm{ET})+0,311^{\star}(\mathrm{S})-0,08396^{\star}(\mathrm{ID})-0,808 \\
\mathrm{~mJ} / \mathrm{d}=0,07885^{\star}(\mathrm{MCM})+0,02132^{*}(\mathrm{MCG})+0,327^{\star}(\mathrm{S})+2,694\end{array}$ & $\begin{array}{l}0,72 \\
0,72\end{array}$ \\
\hline
\end{tabular}

kcal: quilocalorias; mJ: milejoule; kJ: quilojoule; MC: massa corporal; ET: estatura; ID: idade; MCM: massa corporal magra; MCG: massa corporal gorda; S: sexo. 0 valor da TMR foi analisado em valores calóricos por dia. Para realizar a conversão² de mJ/dia, multiplicou-se por 239, e kJ/dia multiplicou-se por 0,239. 
Tabela 2. Distribuição dos sujeitos em relação às variáveis sexo e índice de massa corporal

\begin{tabular}{|c|c|c|c|c|c|c|c|c|c|}
\hline \multirow{3}{*}{ Índice de massa corporal } & \multicolumn{8}{|c|}{ Meninas } & \multirow{3}{*}{ Total } \\
\hline & \multicolumn{8}{|c|}{ Idade } & \\
\hline & 10 & 11 & 12 & 13 & 14 & 15 & 16 & 17 & \\
\hline Baixo peso $\leq 15^{\text {th }}$ & 3 & 1 & 1 & 1 & 3 & 1 & 2 & 1 & 13 \\
\hline Normal $16^{\text {th }}-\leq 85^{\text {th }}$ & 0 & 1 & 8 & 4 & 5 & 6 & 0 & 0 & 24 \\
\hline Sobrepeso $>85^{\text {th }}$ & 1 & 0 & 1 & 1 & 3 & 0 & 0 & 1 & 7 \\
\hline Obeso $>95^{\text {th }}$ & 1 & 1 & 1 & 1 & 1 & 2 & 0 & 0 & 7 \\
\hline Total & 5 & 3 & 11 & 7 & 12 & 9 & 2 & 2 & 51 \\
\hline
\end{tabular}

da medida da bioimpedância (Biodynamics, USA) utilizada somente no cálculo da massa corporal magra e da massa corporal gorda, na equação sugerida por Müller e cols. (13).

Em seguida, realizou-se o procedimento da análise de gases em repouso (Aerosport, TEEM - 100, USA). Registraram-se os valores de minuto em minuto do $\mathrm{VO}_{2}$ e $\mathrm{VCO}_{2}$, em litros por minuto, nos últimos 10 minutos após os 20 minutos de repouso, totalizando 30 minutos de teste. As medidas foram realizadas com o indivíduo em jejum de 6 horas, em posição supina e em repouso muscular (24). Após serem obtidos os valores de $\mathrm{VO}_{2}$ e $\mathrm{VCO}_{2}$, esses foram substituídos na equação de Weir (25), a qual tem sido considerada o método padrão (26):

$\operatorname{TMR}(\mathrm{kcal} / \mathrm{min})=3.942 * \mathrm{VO}_{2}(\mathrm{~L} / \mathrm{min})+$

$$
1.106 * \mathrm{VCO}_{2}(\mathrm{~L} / \mathrm{min})
$$

$\operatorname{TMR}(\mathrm{kcal} / \mathrm{dia})=\operatorname{TMR}(\mathrm{kcal} / \mathrm{min}) * 1.440$

No próximo passo, mediram-se as dobras cutâneas (27) para posterior fracionamento dos componentes corporais em massa corporal magra e em massa corporal gorda. Adotou-se a equação de Lohman (28), com as constantes sugeridas por Pires Neto e Petroski (29).

Percentual de gordura $=1.35^{*}(T R+S E)-0.012$ *

$(T R+S E)^{2}$ - constante

Quando as adolescentes obtiveram a soma dos valores de dobras cutâneas do tríceps e subescapular superiores a $35 \mathrm{~mm}$, utilizou-se a seguinte equação (30):

$\%$ gordura $=0,546 *(\mathrm{TR}+\mathrm{SE})+9,7$

Para transformar o valor de percentual de gordura em valor absoluto, utilizaram-se as seguintes equações:

Massa magra $(\%)=100-\%$ gordura

Massa gorda $(\mathrm{kg})=$ massa corporal * \% gordura 100

Massa magra $(\mathrm{kg})=\underline{\text { massa corporal } * \% \text { Massa magra }}$ 100

Para analisar a validade cruzada das equações de predição (Tabela l) com a medida padrão TMR, realizaram-se os seguintes procedimentos estatísticos: o teste " $\mathrm{t}$ " pareado, para medir a diferença dos valores médios das equações com o valor da TMR padrão, com nível de significância de $\mathrm{p}<0,05$; o erro constante (EC) da medida estimada pela equação com a da TMR mensurada pelo método padrão com critério para aceitar o erro constante uma diferença menor que 5\% (9); e a análise de dispersão gráfica proposta por Bland e Altman (31). Os procedimentos estatísticos foram calculados pelo programa SPSS 11.0 for Windows.

\section{RESULTADOS}

Inicialmente, serão apresentados os resultados das características antropométricas do grupo e, posteriormente, o resultado dos critérios de validação das equações.

O grupo de meninas analisada constituiu-se de 51 adolescentes entre os 10 e 17 anos e suas características antropométricas estão descritas na tabela 3 .

A tabela 4 ilustra os valores médios de consumo de $\mathrm{O}_{2}$ e a produção de $\mathrm{CO}_{2}$ medidos por meio da calorimetria indireta e, posteriormente, utilizados na equação proposta por Weir (25) para o cálculo da TMR.

Tabela 3. Características antropométricas das adolescentes

\begin{tabular}{|c|c|c|}
\hline Variável (unidade de medida) & Média $\pm \mathrm{dp}$ & Amplitude \\
\hline Idade (anos) & $13,2 \pm 1,8$ & $10-17$ \\
\hline Massa corporal $(\mathrm{kg})$ & $51,0 \pm 11,9$ & $26,1-74,6$ \\
\hline Estatura (cm) & $159 \pm 8,5$ & $138-172$ \\
\hline Percentual de gordura ${ }_{D C}(\%)$ & $23,0 \pm 6,0$ & $11,2-34,5$ \\
\hline Massa gorda $_{\mathrm{DC}}(\mathrm{kg})$ & $12,3 \pm 5,6$ & $3,8-24,5$ \\
\hline Percentual de massa magra ${ }_{D C}(\%)$ & $76,9 \pm 6,0$ & $65,4-88,7$ \\
\hline Massa magra $_{\mathrm{DC}}(\mathrm{kg})$ & $38,7 \pm 7,0$ & $22,2-50,3$ \\
\hline Percentual de gordura ${ }_{\mathrm{BIO}}(\%)$ & $24,8 \pm 4,8$ & $18,3-40,3$ \\
\hline Massa gorda $_{\mathrm{B} 10}(\mathrm{~kg})$ & $12,6 \pm 3,6$ & $6,8-23,2$ \\
\hline Percentual de massa magra ${ }_{\mathrm{B} 10}(\%)$ & $75,1 \pm 4,8$ & $59,7-81,7$ \\
\hline Massa magra $_{\text {BIO }}(\mathrm{kg})$ & $38,7 \pm 9,5$ & $55,2-38,3$ \\
\hline
\end{tabular}

DC: medidas obtidas pela técnica de dobras cutâneas; BIO: medidas obtidas pela técnica de bioimpedância; dp: desvio-padrão. 
Tabela 4. Valores do consumo de $\mathrm{O}_{2}$ e produção de $\mathrm{CO}_{2}$ medido pela calorimetria indireta nas adolescentes

\begin{tabular}{lcc}
\hline Variável (unidade de medida) & Média $\pm \mathbf{d p}$ & Amplitude \\
\hline $\mathrm{VO}_{2}(\mathrm{~L} / \mathrm{min})$ & $0,184 \pm 0,048$ & $0,131-0,326$ \\
$\mathrm{VCO}_{2}(\mathrm{~L} / \mathrm{min})$ & $0,154 \pm 0,042$ & $0,104-0,283$ \\
Umidade $(\%)$ & $74,9 \pm 7,4$ & $62-86$ \\
Temperatura $\left(\mathrm{C}^{\circ}\right)$ & $22,8 \pm 2,3$ & $21-26$ \\
\hline
\end{tabular}

dp: desvio-padrão.

A seguir, na tabela 5, estão demonstrados os valores dos critérios adotados para analisar-se a validade das equações femininas.

Tabela 5. Valores dos critérios de validação

\begin{tabular}{lcccc}
\hline Equações & Média $\pm \mathbf{d p}$ & $\begin{array}{c}\text { EC } \\
\text { kcal/dia }\end{array}$ & $\begin{array}{c}\text { EC } \\
\mathbf{\%}\end{array}$ & “t” (p) \\
\hline Weir (25) & $1292 \pm 337$ & - & & - \\
Harris e Benedict (17) & $1375 \pm 122$ & 83,2 & 6,4 & $-1,940(0,058)$ \\
Schofield (18) & $1375 \pm 160$ & 83,6 & 6,4 & $-1,917(0,061)$ \\
WHO/FAO/UNU (19) & $1367 \pm 145$ & 75,7 & 5,8 & $-1,740(0,088)$ \\
Henry e Rees (20) & $1278 \pm 134$ & $-13,4$ & $-1,0$ & $0,310(0,758)$ \\
Molnár e cols. (21) & $1288 \pm 155$ & $-4,0$ & $-0,3$ & $0,084(0,993)$ \\
Tverskaya e cols. (22) & $1425 \pm 250$ & 149,5 & 10,2 & $-3,531(0,001)$ \\
Müller e cols. (13) & $1428 \pm 129$ & 136,1 & 10,5 & $-3,278(0,002)$ \\
Müller e cols. (13) & $1438 \pm 191$ & 162,3 & 11,3 & $-3,798(0,000)$ \\
\hline
\end{tabular}

dp: desvio-padrão; EC: erro constante; "t": teste t; p: significância.

As figuras 1 a 8 ilustram a dispersão dos dados analisados pela proposta de Bland e Altman (31).

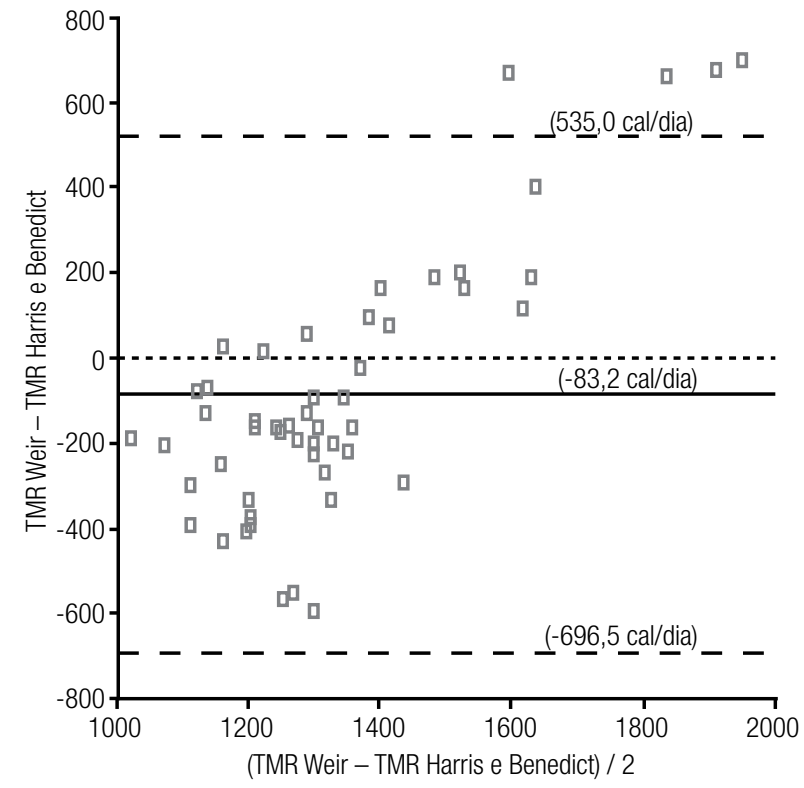

Figura 1. Gráfico de dispersão de Bland e Altman (31) dos valores da equação proposta por Harris e Benedict (17).

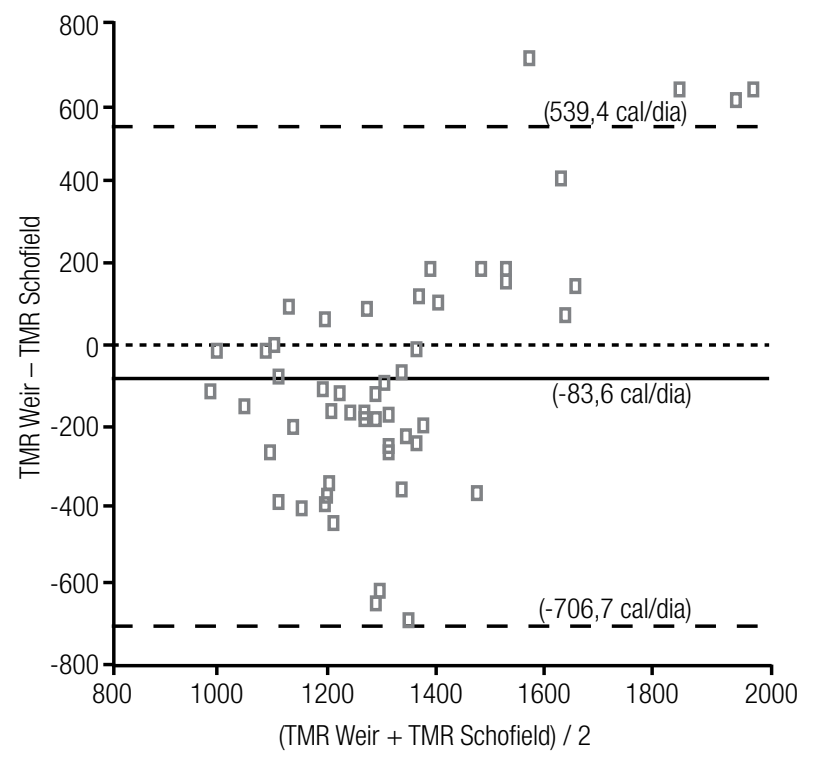

Figura 2. Gráfico de dispersão de Bland e Altman (31) dos valores da equação proposta por Schofield (18).



Figura 3. Gráfico de dispersão de Bland e Altman (31) dos valores da equação proposta por WHO (19).

\section{DISCUSSÃO}

As meninas analisadas neste estudo, quando comparadas com as dos grupos que constituíram o desenvolvimento das equações internacionais, apresentaram valores superiores para a massa corporal e estatura em relação ao grupo utilizado por Schofield (18) e WHO/ $\mathrm{FAO} / \mathrm{UNU}$ (19) e valores inferiores quando comparadas com os grupos analisados por Molnár e cols. (21) e Müller e cols. (13). 


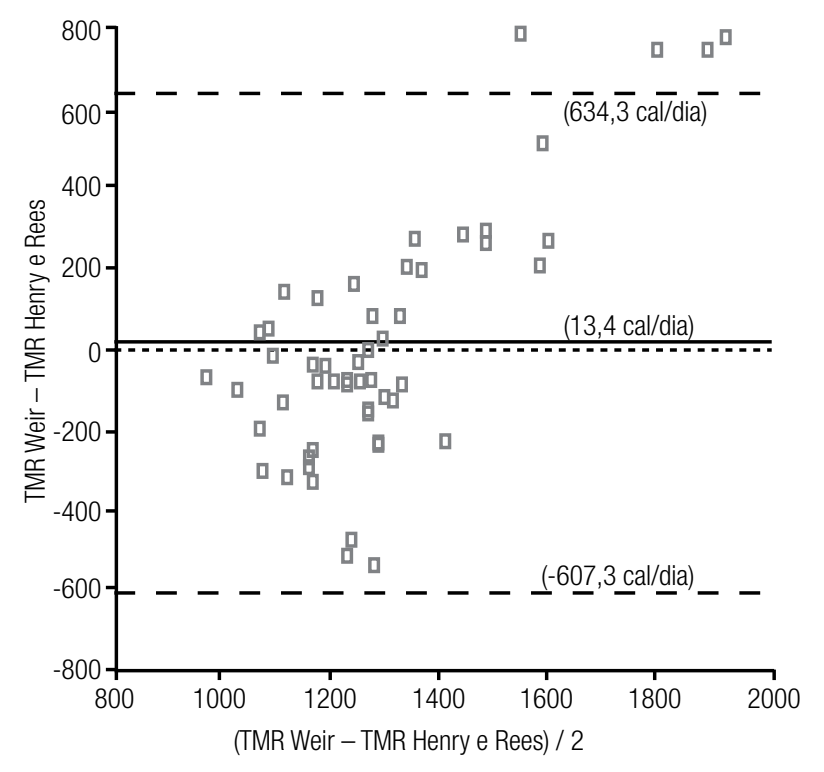

Figura 4. Gráfico de dispersão de Bland e Altman (31) dos valores da equação proposta por Henry e Rees (20).

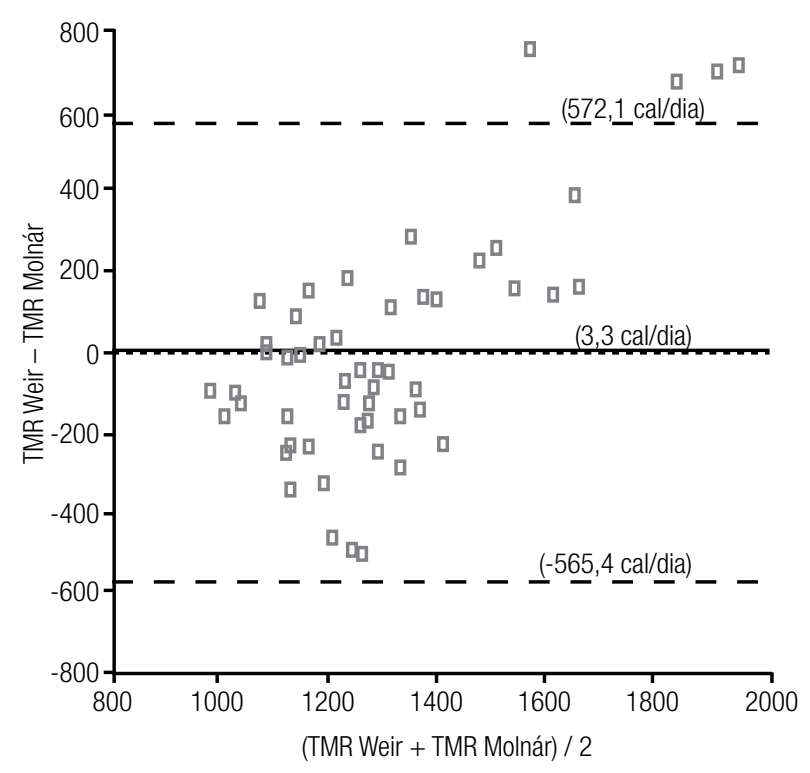

Figura 5. Gráfico de dispersão de Bland e Altman (31) dos valores da equação proposta por Molnár e cols. (21).

Em relação à massa magra, as meninas apresentaram valores similares aos dos trabalhos de Molnár e cols. (21) e Müller e cols. (13).

O grupo analisado por Tverskaya e cols. (22), para todas as medidas, apresentou valores mais elevados do que as meninas deste estudo. Esses valores já eram esperados, pois no estudo foram utilizadas somente adolescentes obesas.

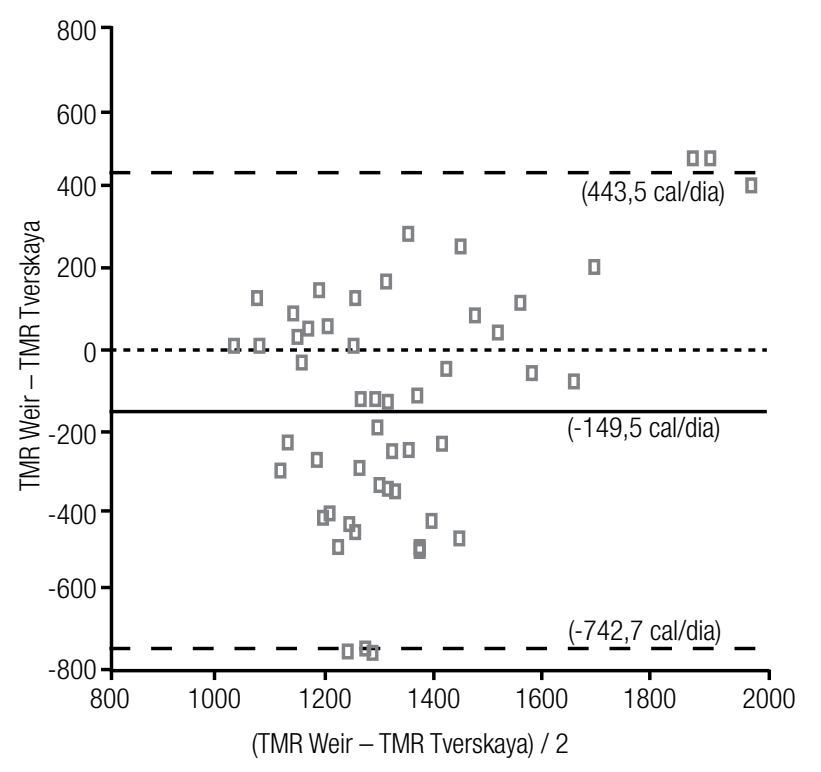

Figura 6. Gráfico de dispersão de Bland e Altman (31) dos valores da equação proposta por Tverskaya e cols. (22).

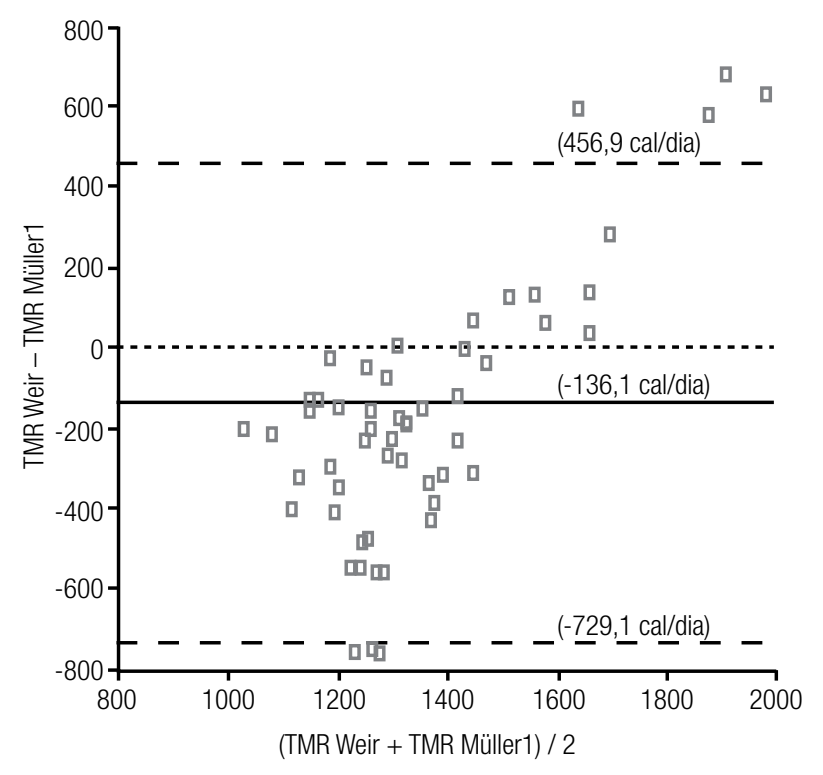

Figura 7. Gráfico de dispersão de Bland e Altman (31) dos valores da equação proposta por Müller e cols. (13).

Como há uma forte relação entre os componentes corporais e os valores da TMR (13), diferenças antropométricas entre a população utilizada para o desenvolvimento das equações que estimam a TMR e a população de adolescentes brasileiras, pode-se justificar possíveis erros das equações.

Analisando-se os critérios adotados para a validação das equações, notou-se que a equação proposta 


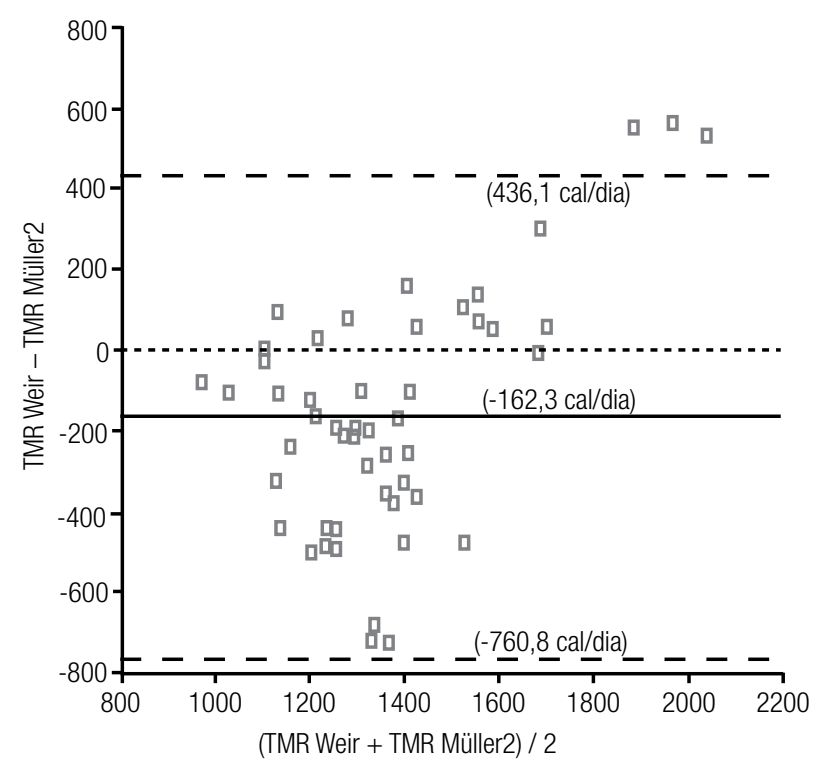

Figura 8. Gráfico de dispersão de Bland e Altman (31) dos valores da equação proposta por Müller e cols. (13).

por Tverskaya e cols. (22) e as equações sugeridas por Müller e cols. (13) foram as únicas que apresentaram diferenças significativas entre as médias.

No entanto, somente as equações propostas por Henry e Rees (20) e Molnár e cols. (21) obtiveram erros constantes inferiores a 5\%. As demais equações, apesar de não terem diferenças estatisticamente, superestimaram os valores da TMR acima de 5\%. Essa tendência já havia sido detectada para as equações de Harris e Benedict $(4,22,32)$, Schofield $(4,20,33)$, $\mathrm{WHO} / \mathrm{FAO} / \mathrm{UNU}(4,21)$ e Tverskaya e cols. (34).

$\mathrm{O}$ estudo revela que, em média, as equações de Harris e Benedict (17), Schofield (18), WHO/FAO/UNU (19), Henry e Rees (20) e Molnár e cols. (21) não se diferenciam do método padrão, porém essas equações não conseguem estimar os valores com igual coerência e magnitude. Isso foi demonstrado por meio da análise dos gráficos de dispersão de Bland e Altman (31). Os gráficos revelam que, independentemente da equação, não há concordância entre as equações e o método padrão.

Esse achado possui reflexo na estimativa da TMR, em ambiente clínico ou de pesquisa, pois os planos de intervenções nutricionais para manutenção, perda ou ganho de peso são estipulados individualmente.

Dessa forma, o estudo indica que nenhuma das equações analisadas alcançou os critérios estipulados, não possuindo, assim, validade nos cálculos da TMR em adolescentes com as características do grupo estudado.
Assim, recomenda-se o desenvolvimento de equações específicas para a população de adolescentes femininas brasileiras.

Agradecimento: Ao Conselho Nacional de Desenvolvimento Científico e Tecnológico $(\mathrm{CNPq})$ pelos recursos financeiros disponibilizados.

Declaração: os autores declaram não haver conflitos de interesse científico neste estudo.

\section{REFERÊNCIAS}

1. Melby $\mathrm{CL}, \mathrm{Ho} \mathrm{RC}$, Hill JO. Avaliação do gasto energético. In: Bouchard C (ed.) Atividade física e obesidade. Barueri: Manole; 2000. p. 117-49.

2. Montoye HJ, Kemper HCG, Saris WHM, Washburn RA. Measuring physical activity and energy expenditure. Champaing: Human Kinetics; 1996.

3. Wang Z, Heshka S, Zhang K, Boozer CN, Heymsfield SB. Resting energy expenditure: systematic organization and critique of prediction methods. Obes Res. 2001;9(5):331-6.

4. Wong WW, Butte NF, Hergenroeder AC, Hill EB, Stuff JE, Smith EO. Are basal metabolic rate prediction equations appropriate for female children and adolescents? J Appl Physiol. 1996;81(6):2407-14.

5. Cruz CM, Silva AF, Anjos LA. A taxa metabólica basal é superestimada pelas equações preditivas em universitárias do Rio de Janeiro, Brasil. Arch Latinoam Nutr. 1999;49(3):233-7.

6. Alfonzo-Gonzales G, Doucet E, Almeras N, Bouchard C,Tremblay A. Estimation of daily energy needs with the FAO/WHO/UNU 1985 procedures in adults: comparison to whole-body indirect calorimetry measurements. Eur J Clin Nutr. 2004;58:1125-31.

7. Garcia ES. Taxa metabólica basal de jogadores profissionais de futebol. Rev Bras Educ Fís Esp. 2006;20:82-4.

8. De Lorenzo A, Bertini I, Puijia A, Testolin G, Testolin C. Comparison between measured and predicted resting metabolic rate in moderately active adolescents. Ital J Neurol Sci. 1999;36:141-5.

9. Frankenfield DC, Roth-Yousey L, Compher C. Comparison of predictive equations for resting metabolic rate in healthy nonobese and obese adults: a systematic review. J Am Diet Assoc. 2005;105(5):775-89.

10. Rocha EEM, Alves VGF, Fonseca RBV. Indirect calorimetry: methodology, instruments and clinical application. Curr Opin Clin Nutr Metab Care. 2006;9:247-56.

11. Thomson MA, Bucolo S, Quirk P, Shepherd RW. Measured versus predicted resting energy expenditure in infants: a need for reappraisal. J Pediatr. 1995;126:21-7.

12. Frankenfield DC, Rowe WA, Smith JS, Cooney RN. Validation of several established equations for resting metabolic rate in obese and nonobese people. J Am Diet Assoc. 2003;103:1152-59.

13. Müller MJ, Bosy-Westphal A, Klaus $S$, Kreymann G, Lührmann PM, Neuhäuser-Berthold $M$, et al. World Health Organization equations have shortcoming for predicting resting energy expenditure in persons from a modern, affluent population: generation of a new reference standard from a undernourishe analysis of a German database of resting energy expenditure. Am J Clin Nutr. 2004;80:1379-90.

14. Schneider P, Meyer F. As equações de predição da taxa metabólica basal são apropriadas para adolescentes com sobrepeso e obesidade? Rev Bras Med Esporte. 2005;11(3):193-7.

15. Wahrlich V, Anjos LA. Validação de equações de predição da taxa metabólica basal em mulheres residentes em Porto Alegre, RS, Brasil. Rev Saúde Pública. 2001;35(1):39-45. 
16. Anjos LA, Braun FLC, Damião JJ, Silva AF. Basal metabolic rate cannot be estimated by current predictive equations in Brazilian college female students. Med Sci Sports Exerc. 1998;30:263.

17. Harris JA, Benedict FG. A biometric study of basal metabolism in man. Washington: Carnegie Institute Publication; 1919.

18. Schofield WN. Predicting basal metabolic rate, new standards and review of previous work. Hum Nutr Clin Nutr. 1985;39:5-41.

19. World Health Organization. Energy and protein requirements. World Health Organization Technical Report Series. Geneva; 1985.

20. Henry CJK, Rees DG. New predictive equations for the estimation of basal metabolic rate in tropical peoples. Eur J Clin Nutr. 1991;45:177-85.

21. Molnár $\mathrm{D}$, Jeges $\mathrm{S}$, Erthardt $\mathrm{E}$, Schutz $\mathrm{Y}$. Measured and predict resting metabolic rate in obese and nonobese adolescents. J Pediatr. 1995; 127:571-77.

22. Tverskaya R, Rising R, Brown D, Lifshitz F. Comparison of several equations an derivation of a new equation for calculating basal metabolic rate in obese children. J Am Coll Nutr. 1998;17(4):333-6.

23. Anjos LA, Veiga GV, Castro IRR. Distribuição dos valores do índice de massa corporal da população brasileira até 25 anos. Rev Panam Salud Pública. 1998;3(3):164-73.

24. Bandini LG, Morelli JA, Must A, Dietz WH. Accuracy of standardized equations for predicting metabolic rate in premenarcheal girls. Am J Clin Nutr. 1995;62:711-14.

25. Weir JBD. New methods for calculating metabolic rate with special reference to protein metabolism. J Physiol. 1949;109:1-9.
26. Wells JCK. Energy metabolism in infants and children. Nutrition. 1998;14(10):817-20.

27. RossWD, Marfell-Jones MT. Kinanthropometry. In: MacDougall JD, Wenger HA, Green HJ, eds. Physiological testing of the high-performance athlete. Champaing: Human Kinetics; 1991. p. 223-308.

28. Lohman TG. Applicability of body composition techniques and constants for children and youth. Exerc Sports Sci Rev. 1986;14:325-57.

29. Pires Neto CS, Petroski E. Assuntos sobre equações da gordura corporal relacionada a crianças e jovens. Comunicação, movimento e mídia na Educação Física. 1996;3:21-30.

30. Slaughter MH, LohmanTG, Boileau CA, Horswill CA, Stillman RJ, Van Loan MD, et al. Skinfold equations for estimation of body fatness in children and youth. Hum Biol. 1988;60:709-23.

31. Bland JM, Altman DG. Statistical methods for assessing agreement between two methods of clinical measurement. Lancet. 1986;12:307-10.

32. Rodrigues $A E$, Marostegan PF, Mancini MC, Dalcanale L, Melo ME, Cercato $C$, et al. Análise da taxa metabólica de repouso avaliada por calorimetria indireta em mulheres obesas com baixa e alta ingestão calórica. Arq Bras Endocrinol Metab. 2008;58(1):76-84.

33. Rodrigues G, Moreno LA, Sarria A, Fleta J, Bueno M. Resting energy expenditure in children and adolescents: agreement between calorimetry and prediction equations. Clin Nutr. 2000;21(3):255-60.

34. Derumeax-Burel H, Meyer M, Morin L, BoirieY. Prediction of resting energy expenditure in a large population of obese children. Am J Clin Nutr. 2004;80:1544-50. 